\title{
Die Europäische Bürgerinitiative: Möglichkeiten und Grenzen eines neuen Elements im EU-Entscheidungssystem
}

\author{
Rudolf Hrbek*
}

\begin{abstract}
Der Vertrag über die Europäische Union (EUV) in der Fassung des Vertrags von Lissabon enthält in Titel II in insgesamt vier Artikeln ${ }^{1}$ „Bestimmungen über die demokratischen Grundsätze“. Art. 11 Abs. 4 EUV führt die Europäische Bürgerinitiative (EBI) als ganz neues Element in das Entscheidungssystem der Europäischen Union ein. Die Institution einer Bürgerinitiative - vielfach auch Bürgerbegehren genannt - gilt als ein direktdemokratisches Instrument und findet sich in den Verfassungsordnungen einer Reihe von EU-Mitgliedstaaten. Es ergänzt dort die Bestimmungen zur repräsentativen Demokratie, während dem Initiativrecht der Stimmbürger in der Schweiz eine das Regierungssystem von Bund, Kantonen und Gemeinden grundlegend prägende Funktion zukommt. ${ }^{2}$

Vorhandensein und Praktizierung direktdemokratischer Instrumente im Rahmen eines Repräsentativsystems werden vielfach sehr positiv bewertet, wobei dies auf der Einschätzung von Partizipation als hoher Wert für ein demokratisches Gemeinwesen beruht. ${ }^{3}$ Solche normativen Erwartungen werden auch an die EBI geknüpft. Die Argumente sind bekannt: direktdemokratische Instrumente seien geeignet, die Legitimation von Entscheidungen zu erhöhen; mehr (direkte) Partizipation sei ein geeignetes Mittel gegen Politikverdrossenheit und Apathie der Bürger; sie fördere die Identifikation der Bürger mit ihrem politischen System. Für die EBI wird in diesem Zusammenhang davon gesprochen, dass ein Mehr an transnationaler Kommunikation, wie es mit entsprechenden Initiativen notwendigerweise verbunden wäre, einen wichtigen Beitrag zur Schaffung eines europäischen öffentlichen Raums leisten würde, eine unerlässliche Voraussetzung für das Entstehen eines europäischen Demos.
\end{abstract}

Dieser Beitrag möchte die Frage erörtern, was von der EBI als einem neuen Element des EU-Entscheidungssystems erwartet werden kann. Dabei geht es nicht um das generelle Pro und Contra in der Einschätzung direktdemokratischer Instrumente ${ }^{4}$ - auch wenn auf einzelne in dieser Grundsatzdiskussion vorgetragenen Argumente zu verweisen sein wird -, sondern um die Prüfung der Frage nach der Bedeutung und möglichen/wahrscheinlichen Wirkung

* Prof. Dr. Rudolf Hrbek, Institut für Politikwissenschaft und Europäisches Zentrum für Föderalismus-Forschung, Universität Tübingen.

1 Artt. 9 bis 12 EUV.

2 Das zeigt sich in der häufig vertretenen Charakterisierung des politischen Systems der Schweiz als Referendumsdemokratie.

3 Jean Paul Jacqué spricht im Titel seines der EBI gewidmeten Editorials im TEPSA Newsletter von ,the birth of participatory democracy“. Vgl. Jean Paul Jacqué: Editorial: State of play of the European Citizens' Initiative (ECI): the birth of participatory democracy, in: Trans European Policy Studies Association: TEPSA NEWSLETTER, Februar 2011, S. 1-2, hier S. 1.

4 Wolfgang Merkel hat sich dazu jüngst in einem kurzen, dabei sehr substanzreichen und ausgewogenen Beitrag geäußert. Auch wenn es in seinem Beitrag im Kern um Volksabstimmungen im Sinne von Entscheidungen, nicht um Initiativen, geht, gelten seine im Ergebnis sehr skeptischen Einschätzungen direktdemokratischer Verfahren, was ihre Wirkung für die demokratische Qualität eines Gemeinwesens und des entsprechenden Politikprozesses betrifft, auch für Volks- und Bürger-Initiativen. Vgl. Wolfgang Merkel: Illusion und Realität, in: Aus Politik und Zeitgeschichte 44-45/2011, S. 47-55. 
der Institution EBI. ${ }^{5}$ Dafür wird zum einen das Regelwerk der EBI - also die entsprechenden Vertragsbestimmungen sowie die Regelungen in der EBI-Verordnung des Europäischen Parlaments und des Rates ${ }^{6}$ - auf den Prüfstand zu legen sein; zum zweiten bedürfen eine Reihe von Rahmenbedingungen für die Anwendung und Wirkung der EBI der Erörterung. Vorab sollen aber zwei Punkte in gebotener Kürze behandelt werden: (1) ein Überblick über frühere Bemühungen und Maßnahmen, die sich auf die Stellung und Rolle des Bürgers im System der Europäischen Gemeinschaft beziehungsweise der Europäischen Union bezogen haben; (2) eine Darstellung der Entstehungsgeschichte der EBI im Verfassungskonvent.

\section{Frühere Bemühungen und Maßnahmen zur Aufwertung des Bürgers im politischen System der EG beziehungsweise EU}

Der 1975 vorgelegte „Bericht über die Europäische Union“ (Tindemans-Bericht) ${ }^{7}$ enthält ein Kapitel IV mit dem Titel „Das Europa der Bürger“. Darin unterbreitete Tindemans eine Reihe von Vorschlägen, „mit denen Europa dem Bürger näher gebracht werden soll“. In Ergänzung zu einer Reihe von in Kapitel III des Berichts („Das wirtschaftliche und soziale Europa“) geforderten Maßnahmen - die sich ,unmittelbar im täglichen Leben der Europäer niederschlagen“ w würden - nannte Leo Tindemans noch Maßnahmen zum Schutz der Rechte der Europäer ,überall dort, wo er nicht mehr ausschließlich von den Mitgliedstaaten gewährleistet werden kann“, nämlich recht allgemein die Anerkennung und den Schutz der Grundrechte und Grundfreiheiten sowie recht konkret Maßnahmen zugunsten der Rechte des Verbrauchers und auf dem Gebiet des Umweltschutzes. Außerdem schlug er Maßnahmen als ,äußere Zeichen unserer Solidarität“ vor, wie etwa die schrittweise Abschaffung der Personenkontrollen an den Grenzen, die Erstattung von Kosten für Heilbehandlungen in anderen Mitgliedstaaten, eine stärkere Verflechtung im Bildungswesen (Austausch von Schülern und Studenten sowie Anerkennung von Abschlüssen) und mehr Kooperation der Hörfunk- und Fernsehanstalten mit dem Ziel besserer gegenseitiger Information.

Bei aller grundsätzlichen Anerkennung der Bemühungen von Tindemans wurde kritisiert, dass der Bericht „über Bürgerrechte schweigt“, obwohl in einem „Europa der Bürger“ auch deren politische Teilhabe rechtlich garantiert werden müsse, ${ }^{8}$ und zwar über das gerade neu beschlossene Wahlrecht zum Europäischen Parlament hinaus. An diese Kritik schloss sich

5 Vgl. dazu auch die relativ frühzeitig, im Oktober 2009 erschienene Studie der Stiftung Wissenschaft und Politik, in der unter anderem auch konkrete Empfehlungen für die Ausgestaltung der EBI-Verordnung gegeben wurden. Vgl. Andreas Maurer/Stephan Vogel: Die Europäische Bürgerinitiative. Chancen, Grenzen und Umsetzungsempfehlungen, Stiftung Wissenschaft und Politik: SWP-Studie, Oktober 2009, S. 1-32, hier S. 28. Ein Jahr später, im November 2010, erschien unter der Verantwortung der Green European Foundation ein umfangreicher Band zu verschiedenen Aspekten der EBI. Vgl. Green European Foundation (Hrsg.): The European Citizens' Initiative Handbook. Your Guide to the World's First Transnational Direct Democracy Tool, Brüssel 2010.

6 Verordnung (EU) Nr. 211/2011 des Europäischen Parlaments und des Rates vom 16. Februar 2011 über die Bürgerinitiative, in: Amtsblatt der EU, Nr. L 65 vom 11. März 2011, S. 1-22 (EBI-Verordnung).

7 Der belgische Ministerpräsident Leo Tindemans hatte diesen Bericht im Auftrag der anderen Staats- und Regierungschefs der EG-Mitgliedstaaten ausgearbeitet. Er sollte einen grundlegenden Beitrag zur Verwirklichung des vom EG-Gipfel 1972 proklamierten, sehr ehrgeizigen Ziels, ,die Gesamtheit der Beziehungen der EG-Staaten in eine Europäische Union zu überführen“, leisten, nämlich den Begriff ,Europäische Union' genauer zu fassen und inhaltlich zu füllen. Vgl. Leo Tindemans: Bericht über die Europäische Union vom 29.12.1975, in: Heinrich Schneider/Wolfgang Wessels (Hrsg.): Auf dem Weg zur Europäischen Union? Diskussionsbeiträge zum Tindemans-Bericht, Bonn 1977, S. 239-288.

8 Eberhard Grabitz: „Die Grundrechte der Europäischen Union. Das Europa der Bürger?“, in: Heinrich Schneider/ Wolfgang Wessels (Hrsg.): Auf dem Weg zur Europäischen Union? Diskussionsbeiträge zum Tindemans-Bericht, Bonn 1977, S. 167-188, hier S. 187. 
die Forderung an, die „Eigenschaft des Bürgers als (National-)Staatsbürger [...] durch ein Europäisches Bürgerrecht“ abzulösen, also ,seine nationalité im Rechtssinne [...] durch eine citoyenneté européenne“ ${ }^{\text {zu }}$ ersetzen. ${ }^{9}$

Ein Jahrzehnt später, im Jahr 1985, legte der Adonnino-Ausschuss - einer der beiden vom Europäischen Rat in Fontainebleau Ende Juni 1984 eingesetzten Ad-hoc-Ausschüsse, ${ }^{10}$ der sich unter dem Vorsitz des Italieners Adonnino mit dem Thema „Europa der Bürger“ befassen und nachfolgenden Gipfeln Bericht erstatten sollte - seinen Bericht vor, ${ }^{1}{ }^{1}$ dessen Vorschlägen der Europäische Rat in Mailand Ende Juni 1985 seine Zustimmung erteilte. Die Arbeit des Ausschusses und das von ihm präsentierte Ergebnis fanden so gut wie gar keine Aufmerksamkeit in Politik und Öffentlichkeit. Sie standen ganz im Schatten der Arbeiten des Dooge-Ausschusses zu den politisch überaus kontroversen institutionellen Fragen. ${ }^{12}$

Der Bericht des Adonnino-Ausschusses enthielt Aussagen und Vorschläge zu einer großen Zahl ganz unterschiedlicher Fragen. Hatte bereits der Europäische Rat von Fontainebleau dem Ausschuss einen recht umfangreichen Themenkatalog vorgegeben, so erweiterte dieser das Themenspektrum noch. Bernd Janssen sprach von einer „Stoffsammlung“ und qualifizierte diese als „Bauchladen““. ${ }^{13}$ Die Bearbeitung des Themenkatalogs in 13 Einzelberichten, für die jeweils ein Ausschussmitglied verantwortlich war, war auf die Zielsetzung ausgerichtet, dass „die vorgeschlagenen Maßnahmen [...] für die Bürger der Gemeinschaft unmittelbare Bedeutung haben und ihnen offensichtlich greifbare Vorteile im Alltag bieten“ sollten. ${ }^{14}$ Viele bezogen sich auf Fragen der Freizügigkeit sowie auf so unterschiedliche Themen wie Kultur und Kommunikation; Information; Jugend, Bildung, Austausch und Sport; freiwillige Entwicklungsarbeit in der Dritten Welt; Gesundheit, Soziale Sicherheit und Drogen; Partnerschaften; sowie Stärkung des Images und der Identität der Gemeinschaft.

Ein weiteres Thema bezog sich explizit auf politische Bürgerrechte. Der Bericht ging damit deutlich über die Aussagen und Anregungen des Tindemans-Berichts hinaus. Die Forderungen zielten auf größere Transparenz der Administration der Europäischen Gemeinschaften (konkret ging es um das Petitionsrecht und die Einrichtung der Institution eines Ombudsman) sowie auf das Recht zu politischer Partizipation von Gemeinschaftsbürgern, die in einem anderen Mitgliedstaat wohnen. Der Ausschuss empfahl für sie die Gewährung des Wahlrechts bei Lokalwahlen, die gleiche Rede- und Versammlungsfreiheit und ein Anhörungsrecht in eigenen Belangen. Weiterhin wurde empfohlen, konsularische Vertretungen in Drittstaaten für alle Bürger der Gemeinschaft nutzbar zu machen.

Vergegenwärtigt man sich, dass im weiteren Verlauf der Gemeinschaftsentwicklung insbesondere durch die Kommission eine ganze Reihe von Anregungen und Empfehlungen des

9 Ebenda, S. 188.

10 Der zweite Ausschuss sollte sich mit Blick auf die Vorbereitung von Vertragsänderungen im Rahmen einer Regierungskonferenz mit institutionellen Fragen befassen. Dieser nach seinem irischen Vorsitzenden James Dooge benannte Dooge-Ausschuss legte dem Europäischen Rat gleichfalls einen Bericht (Dooge-Bericht) vor. Vgl.: European Communities: Report of the Ad Hoc Committee for Institutional Affairs to the European Council. Luxemburg 29.-30. March 1985.

11 Streng genommen handelte es sich um zwei Berichte, die dem Europäischen Rat von Brüssel im März 1985 und dem von Mailand im Juni 1985 unterbreitet wurden. Vgl. Europa der Bürger: Bericht des Ad-hoc-Ausschusses für das Europa der Bürger an den Europäischen Rat, herausgegeben vom Auswärtigen Amt, Bonn 1985 (sogenannter Adonnino-Bericht).

12 So das zweifellos zutreffende Urteil von Bernd Janssen, der in einem Aufsatz deshalb Arbeit und Ergebnis des Ausschusses recht ausführlich beschreibt. Vgl. Bernd Janssen: Das Europa der Bürger - der ,kleine Bruder ‘ im Abseits. Zur Arbeit des Adonnino-Ausschusses, in: integration 4/1985, S. 165-173.

13 Ebenda, S. 166.

14 Ebenda, S. 167. 
Adonnino-Ausschusses aufgegriffen und in Form von Rechtsakten, Programmen und Aktivitäten umgesetzt worden sind, ist es berechtigt, dessen Schlussbericht - wie im Übrigen auch den Tindemans-Bericht - als ein für den Integrationsprozess und die dabei involvierten Akteure wichtiges Referenzdokument zu bezeichnen. Das gilt insbesondere für Initiativen, konkrete Schritte zur Gewährung politischer Bürgerrechte im Primärrecht zu verankern, wie es schließlich im Vertrag von Maastricht erfolgte.

Der Vertrag von Maastricht gilt wegen einer Reihe von Neuerungen - die Einführung der Bezeichnung ,Europäische Union', die ,Pfeiler-Struktur' der Europäischen Union, die Ausweitung der Aufgabenbereiche/Politikfelder der Europäischen Union und institutionelle Reformen - als ein qualitativer Sprung im Integrationsprozess. Dazu gehört auch die Einführung der Unionsbürgerschaft in den Artt. 8 sowie 8a-8e des Vertrags über die Europäische Gemeinschaft (EGV) in der Fassung des Vertrags von Maastricht, in denen erstmals eine Reihe substanzieller Regeln für die Wahrnehmung politischer Bürgerrechte aufgelistet wurden. ${ }^{15}$

Dabei handelt es sich, erstens, um die Gewährung des aktiven und passiven Wahlrechts bei Kommunalwahlen sowie Wahlen zum Europäischen Parlament an Unionsbürger, die ihren Wohnsitz in einem Mitgliedstaat haben, dessen Staatsangehörigkeit sie nicht besitzen. Zweitens handelt es sich um die Gewährung des diplomatischen und konsularischen Schutzes für Unionsbürger in Drittstaaten durch dort bestehende Auslandsvertretungen anderer EUMitgliedstaaten, falls ihr Heimatstaat selbst dort nicht vertreten ist. Drittens schließlich wird bestimmt, dass jeder Unionsbürger das Petitionsrecht beim Europäischen Parlament besitzt und sich an das neu geschaffene Amt des Bürgerbeauftragten (Ombudsman) wenden kann.

Der letzte Artikel zur Unionsbürgerschaft ${ }^{16}$ enthält eine Evolutionsklausel, wonach ergänzende und weiter reichende Bestimmungen erlassen werden können. Die durch den Vertrag von Maastricht neu eingeführten politischen Bürgerrechte werden also nicht als abschließender Katalog verstanden. Die Klausel blieb jedoch zunächst folgenlos. Auch die im Jahr 2000 beschlossene Charta der Grundrechte der Europäischen Union beschränkte sich darauf, die eben genannten konkreten Rechte unter dem Titel V „Bürgerrechte“ nochmals aufzulisten. ${ }^{17}$ Eine Ausweitung um konkrete Partizipationsrechte erfolgte erst mit den Bestimmungen über die EBI.

\section{Die Entstehungsgeschichte der EBI}

Die vom Europäischen Rat in Nizza im Dezember 2000 beschlossene „Erklärung zur Zukunft der Union" "18 wurde als Eingeständnis des - bezogen auf für notwendig angesehene Änderungen und Ergänzungen der vertraglichen Grundlagen der Europäischen Union Scheiterns der Konferenz von Nizza interpretiert. Die Erklärung zur Post-Nizza-Perspektive sah für das Jahr 2004 eine weitere Regierungskonferenz vor und nannte vier konkrete The-

15 Im Vertrag über die Europäische Union in der Fassung des Vertrags von Amsterdam wurden die Bestimmungen zur Unionsbürgerschaft geringfügig ergänzt und finden sich nach der neuen Systematik dort nunmehr unter den Artt. 17-22 EGV. Eine dieser Ergänzungen präzisiert, dass die Unionsbürgerschaft die nationale Staatsbürgerschaft ergänzt, sie aber nicht ersetzt: Art. 8 Abs. 1 EGV in der Fassung des Vertrags von Maastricht beziehungsweise neu Art. 17 Abs. 1 EGV in der Fassung des Vertrags von Amsterdam.

16 Art. 8e EGV in der Fassung des Vertrags von Maastricht beziehungsweise Art. 22 EGV in der Fassung des Vertrags von Nizza.

17 Die Liste wird in der Charta durch zwei Artikel ergänzt, die weitere Rechte betreffen: das „Recht auf eine gute Verwaltung“ sowie das „Recht auf Zugang zu Dokumenten“. Vgl. Artt. 41 und 42 Charta der Grundrechte der Europäischen Union, in: Amtsblatt der EU, Nr. C 303 vom 14. Dezember 2007, S. 1-16.

18 Erklärung zur Zukunft der Union, in: Amtsblatt der EU, Nr. C 80 vom 10. März 2001, S. 85. 
menbereiche für die Diskussion von Vertragsreformen. Den entscheidenden Impuls für die Fortsetzung des Reformprozesses gab der unter belgischer Präsidentschaft stehende Gipfel von Laeken im Dezember 2001. ${ }^{19}$ Er beschloss, erstens, die Einsetzung eines Konvents, der zur Vorbereitung der geplanten Regierungskonferenz über inhaltliche Reformen beraten sollte. Zweitens beschloss der Gipfel, dem Konvent ein umfassendes Mandat zu geben, ihn also nicht auf die vier in Nizza vorgegebenen Punkte zu beschränken. Ausdrücklich wurde als Ziel der „Weg zu einer Verfassung für die europäischen Bürger“20 genannt. Das neue Gremium, das den Beratungen der Regierungskonferenz vorgeschaltet sein sollte, erhielt die Bezeichnung, Verfassungs'-Konvent.

Das inhaltlich weite Mandat für die Ausgestaltung der politischen Grundordnung der künftigen wesentlich erweiterten Europäischen Union und die Zusammensetzung des Konvents mit deutlichem Übergewicht von Parlamentariern gegenüber Repräsentanten der Regierungen erklären, dass in die im März 2002 begonnenen Arbeiten des Konvents teilweise sehr ehrgeizige Vorschläge und Forderungen eingebracht wurden. Dazu gehörten auch Vorschläge zur Einführung direktdemokratischer Entscheidungsformen, die sich erkennbar an Gegebenheiten nicht nur in einzelnen EU-Mitgliedstaaten, sondern vor allem auch der Schweiz orientierten. So wurde ganz offen von der Existenz und Aktivitäten einer „Lobby Suisse“" gesprochen. ${ }^{21}$

Der sicherlich ehrgeizigste Vorschlag sah die Abhaltung eines obligatorischen Referendums über den Vertrag über eine Verfassung für Europa (Verfassungsvertrag) in allen Mitgliedstaaten und am gleichen Tag - gedacht war an den Termin der nächsten Wahl zum Europäischen Parlament, also die Koppelung des Votums über den Vertrag mit der Wahlentscheidung - vor. Hier zeichnete sich letztlich von vornherein ab, dass dieses Verfahren der Vertragsratifizierung nicht realisierbar ist, weil es mit den Bestimmungen der mitgliedstaatlichen Verfassungen unvereinbar war. Andere Vorschläge galten der Aufnahme verschiedener direktdemokratischer Entscheidungsformen in den neuen Vertrag. Sie sollten also nach Inkrafttreten des Verfassungsvertrags Anwendung finden. Dazu gehörte zum einen das Instrument der Bürgerinitiative. Zum zweiten gab es Vorschläge, dass die Institutionen der Europäischen Union den Bürgern bestimmte Fragen zur Entscheidung vorlegen; Gegenstände solcher Referenden sollten Änderungen des Primärrechts sowie andere besonders wichtige Themen - darunter ausdrücklich auch die Entscheidung über die Aufnahme neuer Mitgliedstaaten in die Europäische Union - sein.

Initiatoren dieser und ähnlicher Vorschläge waren ausnahmslos Parlamentsabgeordnete. Besonders exponiert haben sich der Franzose Alain Lamassoure, der Brite Andrew Duff (zusammen mit anderen Liberalen) und der Deutsche Jürgen Meyer. ${ }^{22}$ Das Präsidium des Konvents hat diese Anregungen zunächst allesamt ignoriert und nicht zur weiteren Beratung auf die Tagesordnung gesetzt. Als der Konvent im Juni 2003 die Schlussphase seiner Beratungen begann, ging es um die Formulierung eines Gesamtpakets, welches den Mitgliedern des Konvents vom Präsidium zur generellen Billigung vorgelegt werden sollte. Die Kon-

19 Erklärung von Laeken zur Zukunft der Europäischen Union, in: Europäischer Rat: Schlussfolgerungen des Vorsitzes. Europäischer Rat (Laeken) 14. und 15. Dezember 2001, SN 300/1/01, S. 19-26.

20 Ebenda, S. 23.

21 Über diese und viele andere Interna aus dem Konvent und zur Diskussion um den Themenbereich ,direkte Demokratie' berichtet ein recht prominentes Mitglied des Konvents, der französische Abgeordnete Alain Lamassoure. Vgl. Alain Lamassoure: Histoire secrète de la Convention Européenne, Fondation Robert Schuman/ Albin Michel, Paris 2004, S. 409-425.

22 Ebenda, 409-425. 
ventsmitglieder Lamassoure und Meyer unternahmen dabei gleichsam in letzter Minute den Versuch, wenigstens das Instrument einer Volksinitiative (nach dem Vorbild Italiens und der Schweiz) im neuen Vertrag zu verankern. Sie schlugen vor, dass (mindestens) eine Million Unionsbürger aus acht Mitgliedstaaten eine solche Initiative einbringen können. ${ }^{23} \mathrm{Neun} \mathrm{Ab}$ geordnete des Europäischen Parlaments, 52 Abgeordnete nationaler Parlamente, vier Regierungsvertreter aus Griechenland, Italien, Malta und Rumänien sowie das Präsidiumsmitglied Giuliano Amato unterstützten den Vorschlag. ${ }^{24}$ Auch der Präsident des Konvents, Valéry Giscard d'Estaing, reagierte positiv und übergab das Dossier zur endgültigen Formulierung an den dafür zuständigen Vizepräsidenten, den Belgier Jean-Luc Dehaene. ${ }^{25}$ Das Ergebnis war Art. I-47 Abs. 4 des Verfassungsvertrags. Der Vertrag von Lissabon übernahm die entsprechenden Bestimmungen und führte sie in Art. 11 Abs. 4 EUV ein. Damit war erstmals ein direktdemokratisches Instrument primärrechtlich in das Entscheidungssystem der Europäischen Union aufgenommen, unbeschadet des Umstands, dass es in Art. I-46 Abs. 1 Verfassungsvertrag und in Art. 10 Abs. 1 EUV heißt: „Die Arbeitsweise der Union beruht auf der repräsentativen Demokratie.“ Das Instrument der EBI tritt also nur ergänzend hinzu.

\section{Die Ausgestaltung der EBI}

Der Vertragstext beschreibt zunächst grundlegend den Charakter einer EBI und bestimmt sehr genau, nämlich restriktiv, worauf sich eine solche Initiative inhaltlich beziehen muss. Gemäß Art. 11 Abs. 4 S. 1 EUV darf Gegenstand einer Initiative nur eine Angelegenheit sein, für die die Verträge der Europäischen Union die Kompetenz geben; zu der die Kommission also befugt ist, einen Vorschlag für einen Rechtsakt zu machen, der der Umsetzung der Verträge dient. Initiativen zu Angelegenheiten, die über den von den Verträgen gesetzten Rahmen hinausgehen, sind demnach ausgeschlossen. ${ }^{26}$ Das betrifft auch Initiativen zu Vertragsänderungen. Adressat einer Initiative ist die Kommission, die indessen nicht verpflichtet ist, im Sinn der Initiative tätig zu werden.

Dieser Teil der Vertragsbestimmungen zur EBI lässt einiges offen: Kann man von vornherein davon ausgehen, dass in jedem Einzelfall zweifelsfrei klar ist, ob es sich um einen zulässigen Gegenstand handelt? Wie soll bei Meinungsverschiedenheiten vorgegangen werden? Wie soll die Kommission - auch wenn sie nicht zur Ausarbeitung des Vorschlags für einen Rechtsakt verpflichtet ist - mit einer Initiative umgehen? Auch die Bestimmung, dass es sich bei der mindestens geforderten Zahl von einer Million Unterstützern für eine Initiative

23 Der Vertreter des Deutschen Bundestages, Jürgen Meyer, brachte am 6. Juni 2003 einen entsprechenden Antrag als Ergänzungsvorschlag zu Art. I-46 ein. Vgl. Prof. Dr. Jürgen Meyer, Delegate of the German Bundestag: Amendment Form. Suggestion for amendment of Article: I-46, part I, title VI. (CONV 724/03).

24 Maurer/Vogel: Die Europäische Bürgerinitiative, 2009, S. 9.

25 Lamassoure, auf dessen Darstellung sich die obigen Ausführungen stützen, spricht davon, dass Giscard d'Estaing dieser ,innovation démocratique“ gegenüber ,très favorable“ gewesen sei. Den schließlich in den Verfassungsvertrag aufgenommenen Passus über die EBI bezeichnet er als ,,un joli but marqué à la toute dernière minute“; unverkennbar schreibt er sich den (Haupt-)Verdienst an dieser Lösung zu. Vgl. Lamassoure: Histoire secrète de la Convention Européenne, 2004, S. 425.

26 Vgl. dazu den Wortlaut des Art. 11 Abs. 4 EUV: „Unionsbürgerinnen und Unionsbürger, deren Anzahl mindestens eine Million betragen und bei denen es sich um Staatsangehörige einer erheblichen Anzahl von Mitgliedstaaten handeln muss, können die Initiative ergreifen und die Europäische Kommission auffordern, im Rahmen ihrer Befugnisse geeignete Vorschläge zu unterbreiten, in denen es nach Ansicht jener Bürgerinnen und Bürger eines Rechtsakts der Union bedarf, um die Verträge umzusetzen“. 
um Staatsangehörige „einer erheblichen Anzahl von Mitgliedstaaten handeln muss“ ${ }^{27}$ bedarf näherer Ausformung. Art. 11 Abs. 4 S. 2 EUV schreibt deshalb vor: „Die Verfahren und Bedingungen, die für eine solche Bürgerinitiative gelten, werden nach Artikel 24 Absatz 1 des Vertrags über die Arbeitsweise der Europäischen Union [AEUV] festgelegt." Demnach müssen Europäisches Parlament und Rat - gemäß dem ordentlichen Gesetzgebungsverfahren - eine Verordnung erlassen. ${ }^{28}$

Es dauerte fast eineinhalb Jahre bis die EBI-Verordnung schließlich am 16. Februar 2011 beschlossen wurde. Der lange Entscheidungsprozess erklärt sich aus dem Umstand, dass eine Reihe strittiger Grundsatzfragen sowie komplizierte Verfahrensfragen geklärt werden mussten. Zunächst hatte die Kommission im November 2009 ein Grünbuch verabschiedet ${ }^{29}$ zu dem mehr als 300 Antworten von interessierten Bürgern, Organisationen und Behörden eingingen. Für alle, die sich geäußert hatten, wurde am 22. Februar 2010 in Brüssel eine öffentliche Anhörung durchgeführt. Auf dieser Grundlage formulierte die Kommission einen Vorschlag für eine Verordnung des Europäischen Parlaments und des Rates über die Bürgerinitiative, den sie am 31. März 2010 vorlegte. ${ }^{30}$ Rat und Europäisches Parlament befassten sich in der Folgezeit gemäß dem ordentlichen Gesetzgebungsverfahren - dieses schloss auch die Abgabe von Stellungnahmen des Ausschusses der Regionen (im Juni 2010) und des Wirtschafts- und Sozialausschusses (im Juli 2010) ein - mit dem Entwurf. Die Entscheidung über die 23 Artikel umfassende EBI-Verordnung erfolgte im Februar 2011. Sie gilt ab 1. April 2012.

Die wichtigsten Regelungen der EBI-Verordnung beziehen sich auf folgende Punkte:

- Die Unterzeichner einer Bürgerinitiative müssen aus mindestens einem Viertel der Mitgliedstaaten, gegenwärtig also sieben, kommen. In der Diskussion über diesen Punkt waren auch ein Drittel oder ein Fünftel vorgeschlagen worden.

- Strittig war sodann, welche Mindestanzahl von Bürgern in jedem dieser sieben Mitgliedstaaten als Unterzeichner einer Initiative erforderlich ist. Die Festsetzung eines bestimmten Anteils an der jeweiligen Gesamtbevölkerung - diskutiert wurden hierfür 0,3 Prozent oder 0,2 Prozent - für jeden Mitgliedstaat wurde, mit Blick auf die großen Unterschiede zwischen den Mitgliedstaaten, als Lösung verworfen. Als Quorum für die jeweilige Mindestanzahl wurde schließlich eine Zahl bestimmt, die der Anzahl der im jeweiligen Mitgliedstaat gewählten Mitglieder des Europäischen Parlaments multipliziert mit 750 (der Gesamtzahl der Abgeordneten des Europäischen Parlaments ohne ihren Präsidenten) entspricht. Damit wurde dem Prinzip der degressiven Proportionalität im Verhältnis der Mitgliedstaaten gefolgt. Für Deutschland sind das 74.250, für Luxemburg und Malta 4.500 Bürger.

- Uneinigkeit herrschte zunächst auch hinsichtlich der Frage nach dem Mindestalter der Unterzeichner einer Initiative. Hier standen sich die Befürworter einer Altersgrenze von nur 16 Jahren beziehungsweise von 18 Jahren gegenüber. Die EBI-Verordnung bestimmt diesbezüglich, dass das Mindestalter für die Beteiligung an einer Initiative dem Alter entspricht, das zur Ausübung des aktiven Wahlrechts bei den Wahlen zum Europäischen

27 Art. 11 Abs. 4 S. 1 EUV.

28 Ausdrücklich wird gefordert, dass in der Verordnung auch die „Mindestzahl der Mitgliedstaaten, aus denen die Bürgerinnen und Bürger, die diese Initiative ergreifen, kommen müssen“, festgelegt wird. Vgl. Art. 24 Abs. 1 S. 1 AEUV.

29 Vgl. Europäische Kommission: Grünbuch zur Europäischen Bürgerinitiative, KOM (2009) 622, S. 1-16.

30 Vgl. Europäische Kommission: Vorschlag für eine Verordnung des Europäischen Parlaments und des Rates über die Bürgerinitiative, KOM (2010) 119, S. 1-34. 
Parlament berechtigt. Dieses liegt in allen Mitgliedstaaten bei 18 Jahren mit Ausnahme Österreichs, wo Bürger mit Vollendung des 16. Lebensjahres das aktive Wahlrecht bei den Wahlen zum Europäischen Parlament erhalten.

- Für das Verfahren zur Einleitung einer Bürgerinitiative schreibt die EBI-Verordnung vor, dass die Organisatoren - sie müssen ausnahmslos Unionsbürger sein - einen sogenannten Bürgerausschuss bilden, dem mindestens sieben Personen angehören, die Einwohner von mindestens sieben verschiedenen Mitgliedstaaten sind. Die Organisatoren müssen einen Vertreter und einen Stellvertreter als sogenannte Kontaktpersonen benennen, die als Bindeglied zu den Organen der Union fungieren und im Namen des Bürgerausschusses sprechen und handeln.

- Als nächsten Schritt sieht die EBI-Verordnung vor, dass die Organisatoren eine geplante Initiative bei der Kommission förmlich anmelden. Diese muss innerhalb von zwei Monaten (diskutiert wurde auch über eine doppelt so lange Frist von vier Monaten) prüfen, ob der Gegenstand der Initiative nach den Bestimmungen des Art. 11 Abs. 4 EUV zulässig ist. Kriterien für die Prüfung sind, über den vom Vertrag genannten Rahmen hinaus, dass die geplante Initiative „nicht offenkundig missbräuchlich, unseriös oder schikanös“ ist sowie dass sie ,nicht offenkundig gegen die Werte der Union, wie sie in Artikel 2 EUV festgeschrieben sind“, verstößt. ${ }^{31}$ Fällt die Prüfung positiv aus, wird die Initiative förmlich registriert. Der Grund für diese Verfahrensvorschrift ist, dass bereits vor dem aufwendigen Sammeln von Unterstützungsbekundungen geprüft wird, ob die Initiative zulässig ist. In der Diskussion war auch, dass diese Prüfung erst nach Vorliegen von etwa einem Drittel der insgesamt erforderlichen Unterschriften von einer Million - also etwa 300.000 - und/ oder nach Ablauf eines bestimmten Zeitraums, in dem Unterstützungsbekundungen gesammelt werden, erfolgt.

- Die Organisatoren werden im Übrigen verpflichtet, für das von der Kommission zur Verfügung gestellte Online-Register regelmäßig aktualisierte Informationen über die Quellen der Unterstützung und Finanzierung für die geplante Initiative bereit zu stellen.

- Die Kommission wird verpflichtet, eine Kontaktstelle einzurichten, die insbesondere den Organisatoren Informationen und Hilfe anbietet.

- Nach erfolgter Registrierung kann die Sammlung der Unterstützungsbekundungen beginnen, für die ein Zeitraum von zwölf Monaten vorgesehen ist. Die Bekundungen können in Papierform oder elektronisch abgegeben werden. Die Prüfung, ob die Unterstützer abstimmungsberechtigt waren, wird von den jeweils zuständigen Behörden des betreffenden Mitgliedstaats vorgenommen. Diese legen auch die Kriterien für die Anerkennung der Abstimmungsberechtigung fest. Die Prüfung muss innerhalb von maximal drei Monaten abgeschlossen sein. Den Organisatoren wird eine entsprechende Bescheinigung ausgestellt.

- Erst dann können die Organisatoren die Bürgerinitiative bei der Kommission vorlegen. Sie müssen ergänzend auch „Informationen über jedwede Unterstützung und Finanzierung der gesamten Initiative der Kommission vorlegen." Überschreitet die aus einer einzigen Quelle stammende finanzielle Unterstützung einen bestimmten Umfang, muss diese Quelle angegeben werden. Dieser Umfang entspricht dem in den Regelungen für die politischen

31 Diese Bestimmungen zeigen deutlich, dass einer Instrumentalisierung und einem Missbrauch der Institution EBI von vornherein ein Riegel vorgeschoben werden soll. 
Parteien auf europäischer Ebene und ihre Finanzierung festgelegten Umfang und beträgt 500 Euro. ${ }^{32}$

- Geht bei der Kommission eine Bürgerinitiative samt begleitenden Dokumenten ein, veröffentlicht diese sie im Register und gibt den Organisatoren Gelegenheit, ihr die mit der Initiative angesprochenen Aspekte detailliert zu erläutern. Das ist als zusätzliche Entscheidungsgrundlage für die Kommission gedacht.

- Die Kommission ist gemäß den Vertragsbestimmungen nicht verpflichtet, der Initiative zu folgen. Die EBI-Verordnung verpflichtet sie lediglich, innerhalb von drei Monaten in einer förmlichen Mitteilung - sie wird den Organisatoren, dem Europäischen Parlament und dem Rat übermittelt und veröffentlicht - ihre rechtlichen und politischen Schlussfolgerungen, ihr weiteres Vorgehen beziehungsweise den Verzicht auf ein weiteres Vorgehen und die Gründe dafür darzulegen.

- Im Fall einer erfolgreichen Initiative wird den Organisatoren die Möglichkeit gegeben, die Initiative im Rahmen einer öffentlichen Anhörung vorzustellen. Für die Organisation dieser Anhörung, die im Europäischen Parlament stattfindet, sind das Europäische Parlament und die Kommission gemeinsam zuständig. Das beinhaltet, auch anderen Organen und Einrichtungen der Union die Teilnahme zu ermöglichen.

Die oben gegebene Übersicht über die Vertragsbestimmungen und die ergänzende, sehr detaillierte EBI-Verordnung zeigen, dass die Nutzung des neuen Instruments der EBI in einem genau reglementierten und recht komplexen Verfahren erfolgen muss, das insbesondere die Initiatoren vor eine Vielzahl politischer und vor allem organisatorischer Herausforderungen stellt. Ob und inwieweit diese bewältigt werden können, hängt auch von einer Reihe von Rahmenbedingungen ab. Was für die Anwendung und mögliche Wirkung des neuen direktdemokratischen Instruments der EBI erwartet werden kann, wird stark von solchen Herausforderungen und Rahmenbedingungen sowie der Frage, wie politische Akteure - also die Organisatoren von entsprechenden Initiativen - mit ihnen umgehen, abhängen.

\section{Überlegungen zur Nutzung und möglichen Wirkung des neuen Instruments der EBI}

Die an die EBI geknüpften normativen Erwartungen sind bereits in der Einleitung dieses Beitrags wie folgt zusammengefasst: direktdemokratische Instrumente, also auch die EBI, seien geeignet, die Legitimation von Entscheidungen zu erhöhen; mehr (direkte) Partizipation sei ein geeignetes Mittel gegen Politikverdrossenheit und Apathie der Bürger; sie fördere die Identifikation der Bürger mit ihrem politischen System. Und: da mit der EBI mehr transnationale Kommunikation und Vernetzung verbunden wäre, würde sie einen wichtigen Beitrag zur Schaffung eines europäischen öffentlichen Raumes leisten, was wiederum eine unerlässliche Voraussetzung für das Entstehen eines europäischen Demos sei. Diese Erwartungen sind von recht allgemeiner Art und es muss deshalb geprüft und erörtert werden, ob die EBI sie realisieren kann. ${ }^{33}$

32 Vgl. Art. 6 Lit. b Verordnung (EG) Nr. 2004/2003 des Europäischen Parlaments und des Rates vom 4. November 2003 über die Regelungen für die politischen Parteien auf europäischer Ebene und ihre Finanzierung, in: Amtsblatt der EU, Nr. L 297 vom 15. November 2003, S. 1-4.

33 Maurer und Vogel haben bereits vor Beginn der Diskussion um die EBI-Verordnung auf Chancen, aber eben auch auf Grenzen, also restriktive Faktoren, für die Wirksamkeit der EBI hingewiesen. Vgl. Maurer/Vogel: Die Europäische Bürgerinitiative, 2009. 
Adressaten, potenzielle Initiatoren, restriktive Faktoren: Grenzen der Reichweite des neuen Instruments

Verfolgt man die Diskussion um die EBI, so fällt zunächst auf, dass nicht argumentiert wird, die Nutzung der neuen Initiative sei nötig oder auch nur geeignet, inhaltlich bessere Politik-Entscheidungen herbeizuführen. Der Hinweis auf, output'-Legitimität fehlt. Der EBI wird also ganz offensichtlich nur eine Funktion bei der Schaffung von ,input'-Legitimität zugeschrieben. Das ist insofern nicht überraschend, weil auf der Tagesordnung der Europäischen Union eine große Zahl von Politik-Projekten steht und die verschiedensten Akteure - EU-Institutionen, nationale Regierungen und Lobbyisten jedweder Art (etablierte Interessenverbände, Nicht-Regierungsorganisationen, ,Zivilgesellschaft") - darauf hinwirken, diese Tagesordnung zu erweitern. Mit der EBI soll demnach nur ein zusätzlicher Akteur am Agenda-Setting mitwirken.

Diese Mitwirkung ist zweifellos mehr als bloße Konsultation, zu der sich die Kommission mit ihrem Weißbuch „Europäisches Regieren“34 aus dem Jahr 2001 ganz generell offiziell verpflichtet hat, gerade auch gegenüber Akteuren der Zivilgesellschaft. Die EBI-Verordnung gestaltet diese aber zu einem bis in Einzelheiten streng formalisierten Instrument, entspricht also nicht der Empfehlung, ,,initiatorenfreundlich“" ${ }^{35}$ zu sein. Initiatoren werden genau darauf achten müssen, allen formalen Anforderungen zu genügen, was aufwendig ist und organisatorische Fähigkeiten und Erfahrungen, nicht zuletzt auch finanzielle Ressourcen, verlangt.

Mit einer Bürgerinitiative ist ganz generell die Vorstellung verbunden, dass der einzelne Bürger Zugang zum politischen Prozess erhalten soll und sich eigenständig einbringen kann. Erfahrungen mit der praktischen Nutzung des Instruments der Bürgerinitiative in nationalen politischen Systemen zeigen, dass es letztlich etablierte politische Organisationen - Parteien, Interessenverbände und neuerdings auch zivilgesellschaftliche Organisationen - sind, die die Nutzung des Instruments dominieren. Sie verfügen über eine Mitgliederbasis, eingespielte Organisationsstrukturen und finanzielle Ressourcen als Voraussetzungen für Kampagnenfähigkeit. Ergänzend kommen Kommunikationsbeziehungen (Vernetzung) mit anderen Organisationen und Zugang zu Medien und der Öffentlichkeit hinzu. Und selbst in Fällen, in denen ein spezifisches Anliegen in einer sich spontan gebildeten Bürgerbewegung wurzelt, benötigt diese, um das Anliegen mit dem förmlichen Instrument einer Bürgerinitiative voran zu bringen, einer organisatorischen Infrastruktur und der Unterstützung durch etablierte Organisationen. Das wird auch für die Nutzung der EBI gelten.

Etablierte politische Organisationen könnten sich allerdings fragen, ob sie ein spezifisches eigenes Anliegen nicht auf vielfach erprobte Weise in den Entscheidungsprozess einbringen können, statt sich auf das nicht nur stark formalisierte, sondern vor allem auch langwierige Verfahren der neuen EBI einzulassen. Was den Zeitfaktor betrifft: von der Bildung eines Bürgerausschusses bis zur Entscheidung der Kommission über die ihr vorgelegte EBI vergehen etwa eineinhalb Jahre und erst danach würde - gegebenenfalls - ein Gesetzgebungsverfahren mit der Unterbreitung einer Kommissionsvorlage beginnen. Für ein von der Sache her als dringlich angesehenes Anliegen wäre das Zeitverschwendung. Die Nutzung der EBI macht demnach nur insofern Sinn, wenn es den Initiatoren um den Anstoß zu einem Kommunikations- und Lernprozess geht.

34 Europäische Kommission: Europäisches Regieren. Ein Weissbuch, KOM (2001) 428.

35 Vgl. Maurer/Vogel: Die Europäische Bürgerinitiative, 2009, S. 16. 
Ein anderer Gesichtspunkt gilt dem Umstand, dass eine EBI zunächst immer nur das Anliegen einer Minderheit ausdrückt, wobei offen bleibt, was die große Mehrheit der Unionsbürger davon hält. Die Repräsentativität eines von einer Minderheit per EBI artikulierten Petitums kann durchaus hoch sein. Nur bedarf es dann aber des aufwendigen und langwierigen Verfahrens einer EBI?

Das verweist auf einen anderen Aspekt. Eine, möglicherweise gut organisierte und insofern schlagkräftige, Minderheit könnte versuchen, über den Weg einer EBI Aufmerksamkeit für sich zu gewinnen, PR in eigener Sache zu betreiben. Eine Instrumentalisierung würde auch vorliegen, wenn eine solche Minderheit die EBI in populistischer Weise zu nutzen versucht, vielleicht mit der Absicht, mit der Thematisierung eines Anliegens zu polarisieren beziehungsweise eine bereits virulente Polarisierung noch zu steigern.

Eine EBI zielt stets auf eine Einzelmaßnahme und hat nicht das Potenzial, einen Politikoder einen Paradigmen-Wechsel herbeizuführen oder wenigstens wirksam zu fördern. Um Einzelmaßnahmen zu realisieren, bietet das EU-Entscheidungssystem eine Vielzahl erprobter und den politischen Akteuren wohl vertrauter Instrumente und Strategien. Vor diesem Hintergrund ist fraglich, was eine EBI demgegenüber an Innovationspotenzial besitzt. Vor allem etablierten Organisationen stellt sich deshalb die Frage, ob sie mit Blick auf eine solche Einzelmaßnahme den ,Umweg` über eine EBI benötigen oder warum sie ihn vorziehen sollten. Und wenn sich eine Minderheit anschickt, eine Initiative anzustoßen, wird sie in aller Regel auf Bundesgenossen, also die Unterstützung durch starke - nicht zuletzt auch finanzstarke - Organisationen angewiesen sein. Die Initiative ist dann nicht in erster Linie ein Instrument für den einzelnen Bürger, sondern gibt den ohnehin im politischen Prozess privilegierten und dominierenden Kräften - sie werden vielfach als ,die Eliten' apostrophiert und als solche kritisiert - ein zusätzliches Instrument in die Hand. ${ }^{36}$

Könnte eine EBI die Funktion haben, Präferenzen einzelner EU-Institutionen zu unterstützen und ihnen (zusätzliche) Legitimität zu geben? Die Kommission wird bei der Wahrnehmung ihres Initiativrechts stets überlegen, ob eine entsprechende Initiative Erfolgschancen hat und wird sich nicht auf aussichtslose Vorhaben einlassen. Sie kann im Vorfeld eines von ihr einzuleitenden Rechtsetzungs-Verfahrens Diskussionen (etwa durch Vorlage eines Weißbuchs) anstoßen und mittels förmlicher Anhörungen das Ausmaß an Resonanz erkunden beziehungsweise um positive Resonanz werben. Das Europäische Parlament hat ein Aufforderungsrecht gegenüber der Kommission und kann, wenn sich entsprechende Mehrheiten bilden, mit Initiativberichten auf die Einleitung eines Rechtsetzungsverfahrens hinwirken. Beide, Kommission und Europäisches Parlament, bedürfen also nicht der Unterstützung einer EBI, zumal diese als ein Instrument in den Diensten einer Minderheit von Unionsbürgern ausgestaltet ist.

\section{Innovationspotenzial für transnationale Kommunikationsprozesse}

Das wirklich Neue und Innovative, das mit der EBI verbunden ist, liegt darin, dass die Nutzung des neuen Instruments einen transnationalen Kommunikationsprozess voraussetzt

36 Merkel spricht in diesem Zusammenhang auch von „sozialer Selektivität“. Er schreibt: „Volksabstimmungen gewähren dem Volk tatsächlich mehr Mitwirkungsmöglichkeiten, aber sie verstärken die Tendenz der Überrepräsentation jener gut situierter Schichten, die schon in den Organisationen und Institutionen der repräsentativen Demokratie überproportional vertreten sind.“ Und an anderer Stelle verweist er auf den ,asymmetrisch privilegierten Einfluss“, den mächtige Interessenverbände und multinationale Unternehmen, weil sie über Macht und Geld verfügen, haben. Vgl. Merkel: Illusion und Realität, 2009, S. 50-52. 
beziehungsweise anstößt, wie er bislang nicht existierte und vielleicht auch (noch) nicht nötig war. Als während der Beratungen im Verfassungskonvent über die vertragliche Verankerung direktdemokratischer Instrumente diskutiert wurde, konstituierte sich ein „Initiative and Referendum Institute Europe (IRI Europe)“ als eine Plattform für Überlegungen zu ganz praktischen Aspekten für die spätere Nutzung solcher Instrumente. Das IRI Europe war in den Jahren 2005 bis 2008, konkret bezogen auf die EBI nach dem Verfassungsvertrag, sehr aktiv. Es wurden jährliche ,EBI-Gipfel' abgehalten und die Ergebnisse dieser Überlegungen wurden in jährlich erscheinenden „Initiative for Europe-Handbüchern“ publiziert, die als ganz konkrete Handreichungen für die Nutzung des Instruments einer EBI zu verstehen sind. ${ }^{37}$ Insgesamt 25 in den Jahren 2004 bis 2010 durchgeführte Pilotprojekte für die EBI lieferten den Verfassern der Handreichungen reichhaltiges Erfahrungsmaterial. ${ }^{38}$

Was den transnationalen Kommunikationsprozess betrifft, so müssen sich bereits für den Start einer EBI, nämlich die Bildung eines Bürgerausschusses, Akteure aus mindestens sieben Mitgliedstaaten verständigen und zusammenfinden. Bei diesen Akteuren wird es sich nicht um Unionsbürger als einzeln und isoliert agierende Individuen handeln, sondern um Unionsbürger in ihrer Eigenschaft als Angehörige von Organisationen, in denen sie Leitungsfunktionen innehaben. Erforderlich ist zudem, dass sie sich der Unterstützung für eine Initiative seitens eines möglichst großen Teils ihrer jeweiligen Organisation sicher sein können. Nur mit einem solchen sicheren und verlässlichen Stimmenpaket werden sie Partner in anderen Mitgliedstaaten gewinnen können. Ferner sollte eine finanzielle Grundausstattung vorhanden sein. ${ }^{39} \mathrm{Zu}$ der Zeitspanne von etwa eineinhalb Jahren für die Durchführung der EBI kommt also noch ein zusätzlicher Zeitraum hinzu.

Transnationale Kommunikation ist auch während der weiteren Stadien der Durchführung einer EBI erforderlich; nicht in erster Linie für die Sammlung der erforderlichen Zahl von Unterschriften in mindestens sieben Mitgliedstaaten - diese Aufgabe kann im jeweiligen nationalen (und regionalen) Rahmen je für sich durchgeführt werden. Sehr wohl aber für Planung und Durchführung von Aktivitäten zur Erläuterung und Rechtfertigung der Initiative gegenüber Medien, anderen Organisationen und der Öffentlichkeit, um den europäischen Charakter der Initiative herauszustreichen und ein den Erfolg der Initiative förderndes Meinungsklima herzustellen. Weiterhin auch für die Zusammenstellung der Informationen über Unterstützung und Finanzierung, die die Initiatoren gemeinsam der Kommission vorlegen müssen und die dann im Online-Register veröffentlicht werden. Schließlich auch für die Vorbereitung des Auftritts der Initiatoren bei der öffentlichen Anhörung, sofern die Initiative erfolgreich war.

Als Träger und Hauptakteure dieser transnationalen Kommunikation kommen in erster Linie Organisationen infrage, die auch im politischen Prozess ganz allgemein eine Rolle spielen; also etablierte Interessenverbände (wie etwa Gewerkschaften), Kirchen, Organisationen der Zivilgesellschaft einschließlich von NGOs, aber auch sektorale Wirtschaftsver-

37 Über die IRI Europe informiert recht ausführlich das von Kaufmann verfasste Handbuch. Vgl. Green European Foundation: The European Citizens' Initiative Handbook, 2010, insbesondere S. 54-73.

38 Eine Übersicht über diese Pilotprojekte enthält das zuvor genannte Handbuch. Ebenda, S. 63-66.

39 Das IRI Europe Handbuch 2008 listet insgesamt vier ,,decisive launching criteria“ auf, ,, which concern the aspect of alliance building at regional and local level, the support of NGO's, the commitment to gather signatures and the financial resources to be fundraised."Nur wenn diese Kriterien erfüllt seien, sollte und könnte eine Kampagne zur Gewinnung von einer Million Unterschriften gestartet werden. Hier werden mindestens 100.000 Unterschriften und ein Betrag von 100.000 Euro genannt. Vgl. Green European Foundation: The European Citizens' Initiative Handbook, 2010, S. 63. 
bände. Sie alle finden sich unter den Initiatoren der 25 oben erwähnten Pilotprojekte. Diese Übersicht verweist auf einen weiteren Aspekt. Es handelt sich vielfach um transnationale, also EU-weit agierende Organisationen, denen als europäischen Dachverbänden nationale Verbände und Organisationen als Mitglieder angehören. Transnationale Organisationen, die über ein gewisses Maß an organisatorischer Infrastruktur und an Erfahrung mit transnationaler Kommunikation (,networking') verfügen, werden bei der künftigen Nutzung des Instruments EBI eine wichtige Rolle spielen. Mitgliederstärke und finanzielle Ressourcen sind zusätzliche Faktoren.

Die Sprachenvielfalt in der Europäischen Union ist ein Faktor, dem für transnationale Kommunikation - vor allem, wenn diese nicht auf den kleinen Kreis des Führungspersonals, der Funktionäre, der jeweiligen Organisation beschränkt sein soll - großes Gewicht zukommt. Bisherige Erfahrungen mit den Wahlkämpfen zum Europäischen Parlament zeigen, dass transnationale Kampagnen so gut wie nicht stattgefunden haben, was nicht nur, aber auch auf Sprachbarrieren zurückgeführt werden muss. Auch diesbezüglich ist der Weg bis zur Existenz eines europäischen öffentlichen Raums weit und mühsam.

$\mathrm{Zu}$ den Trägern transnationaler Kommunikation wird man auch politische Parteien und zwar vor allem „Parteien auf europäischer Ebene“, wie sie seit ihrer erstmaligen Erwähnung im Vertrag von Maastricht genannt werden, ${ }^{40}$ zählen müssen. Diese europaweiten ParteiOrganisationen, deren Hauptaufgabe im Bereich transnationaler politischer Kommunikation liegt, haben sich seit ihrer Gründung Mitte der 1970er Jahre im Vorfeld der ersten europäischen Direktwahlen, was Organisation und politische Kohäsion angeht, weiter entwickelt ${ }^{41}$ und ihre Zahl ist von zunächst drei, dann fünf, auf seit Inkrafttreten des europäischen Parteienstatuts $2003^{42}$ gegenwärtig elf angestiegen. Der Zugang zu Haushaltsmitteln der Europäischen Union, also die Einführung der Parteienfinanzierung - nach nationalen Vorbildern - auf EU-Ebene, ist zweifellos ausschlaggebend für dieses Wachstum in der europäischen Parteienlandschaft gewesen. ${ }^{43}$

In einer ersten Revision des europäischen Parteienstatuts im Jahr $2007^{44}$ wurden Politische Stiftungen auf europäischer Ebene ${ }^{45}$ als weitere Zuwendungsempfänger aus Mitteln des EUHaushalts genannt. Neben Parteien auf europäischer Ebene existieren, ihnen angeschlossen,

40 Art. 138a EGV in der Fassung des Vertrags von Maastricht lautete: „Politische Parteien auf europäischer Ebene sind wichtig als Faktor der Integration in der Union. Sie tragen dazu bei, ein europäisches Bewusstsein herauszubilden und den politischen Willen der Bürger der Union zum Ausdruck zu bringen.“ Im Vertrag von Amsterdam enthielt Art. 191 EGV den identischen Wortlaut. Art. I-46 Abs. 4 Verfassungsvertrag formulierte etwas kürzer wie folgt: „Politische Parteien auf europäischer Ebene tragen zur Herausbildung eines europäischen politischen Bewusstseins und zum Ausdruck des Willens der Bürgerinnen und Bürger der Union bei.“ Der EUV in der Fassung des Vertrags von Lissabon enthält die identische Formulierung in Art. 10 Abs. 4.

41 Vgl.: Jürgen Mittag (Hrsg.): Politische Parteien und europäische Integration. Entwicklung und Perspektiven transnationaler Parteienkooperation in Europa, Essen 2006. Der Sammelband informiert mit 32 Einzelbeiträgen detailliert über die verschiedensten Aspekte dieser Entwicklung.

42 Es handelt sich um die Verordnung (EG) Nr. 2004/2003.

43 Pro Jahr werden insgesamt etwa 15 Millionen Euro nach einem bestimmten Schlüssel (Hauptkriterium ist die Zahl der Abgeordneten im Europäischen Parlament der entsprechenden Partei) auf die einzelnen Parteien verteilt.

44 Verordnung (EG) Nr. 1524/2007 des Europäischen Parlaments und des Rates vom 18. Dezember 2007 zur Änderung der Verordnung (EG) Nr. 2004/2003 über die Regelungen für die politischen Parteien auf europäischer Ebene und ihre Finanzierung, in: Amtsblatt der EU, Nr. L 343 vom 27. Dezember 2007, S. 5-8.

45 Diese sind definiert als „Einrichtungen, die [...] einer politischen Partei auf europäischer Ebene angeschlossen sind und durch ihre Arbeit [...] die Ziele dieser politischen Partei auf europäischer Ebene unterstützen“. Vgl. Art. 1 der Verordnung (EG) Nr. 1524/2007. 
mittlerweile die entsprechenden europäischen Stiftungen. ${ }^{46}$ Die Auflistung der Aufgaben, die die Stiftungen gemäß der Verordnung über die Regelungen für die politischen Parteien auf europäischer Ebene und ihre Finanzierung vor allem wahrnehmen sollen, ergibt, dass Aktivitäten im Zusammenhang mit Vorbereitung und Durchführung von Europäischen Bürgerinitiativen gerade auch von diesen Stiftungen erwartet werden können. Art. 1 jener neuen Verordnung nennt folgende vier Aufgabenbereiche: „Beobachtung, Analyse und Bereicherung von Diskussionen über Themen der europäischen Politik und den Prozess der europäischen Integration; Entwicklung von Tätigkeiten in Verbindung mit europäischen Themen wie zum Beispiel die Durchführung oder Unterstützung von Seminaren, Fortbildungsmaßnahmen, Konferenzen und Studien zu diesen Themen unter Mitwirkung einschlägiger Akteure einschließlich Jugendorganisationen und sonstiger Vertreter der Zivilgesellschaft; Entwicklung der Zusammenarbeit mit gleichartigen Einrichtungen, um die Demokratie zu fördern; Schaffung einer Plattform für die Zusammenarbeit auf europäischer Ebene von nationalen politischen Stiftungen, Wissenschaftlern und anderen einschlägigen Akteuren.“

Sowohl Parteien auf europäischer Ebene als auch die ihnen angeschlossenen Stiftungen kommen demnach als Träger transnationaler Kommunikationsprozesse im Zusammenhang mit der Nutzung des Instruments der EBI infrage.$^{47}$ Es bleibt abzuwarten, ob sie es tun und in welchem Ausmaß sie sich engagieren. Kleinere Parteien auf europäischer Ebene, deren Vertretung im Europäischen Parlament entsprechend bescheiden ist und deren politische Gestaltungsmöglichkeiten im Rahmen des fest institutionalisierten ,normalen“ Entscheidungsprozesses gering sind, könnten die EBI eher als willkommenen Rahmen ansehen und nutzen, ihre (Minderheiten-)Positionen einzubringen und zu propagieren, als ihre größeren Konkurrenten. Aber auch für diese kann es gute Gründe für aktives Engagement geben.

Die oben erwähnten 25 Pilotprojekte zeigen, dass als Träger einer EBI in erster Linie Organisationen infrage kommen, die dem Bereich von etablierten Interessenverbänden, von NGOs und der Zivilgesellschaft zuzurechnen sind. Inwieweit zwischen einzelnen von ihnen und einzelnen Parteien auf europäischer Ebene und ihren Stiftungen Allianzen gebildet werden, bleibt abzuwarten. Ein durchgängiges Muster ist zunächst nicht zu erwarten.

Wenn nach möglichen Trägern - oder wenigstens unterstützenden Mit-Trägern - für eine EBI gefragt wird, kann auch an nationale (gegebenenfalls auch regionale) Parlamente gedacht werden. Art. 12 EUV in Verbindung mit dem Protokoll über die Rolle der nationalen Parlamente in der Europäischen Union ${ }^{48}$ und dem Protokoll über die Anwendung der Grundsätze der Subsidiarität und der Verhältnismäßigkeit ${ }^{49}$ macht sie zu einem neuen zusätzlichen institutionellen Akteur im EU-Entscheidungssystem. Titel II des Protokolls über die Rolle der nationalen Parlamente zielt auf die Förderung einer Zusammenarbeit zwischen den Parlamenten. Um die Möglichkeiten bei der Kontrolle der Einhaltung des Subsidiaritätsprinzips auszuschöpfen, ist intensive Kooperation zwischen nationalen Parlamenten erforderlich. Bei dieser transnationalen Kommunikation entwickelte Praktiken und die entsprechenden Er-

46 Pro Jahr werden insgesamt knapp zehn Millionen Euro nach einem bestimmten Schlüssel (Hauptkriterium ist ebenfalls die Zahl der Abgeordneten im Europäischen Parlament der entsprechenden Partei) auf die einzelnen Stiftungen verteilt.

47 Die Green European Foundation gehört beispielsweise zu den Sponsoren des Handbuchs zur EBI und dokumentiert damit ihre Absicht, sich bei der Nutzung des neuen Instruments zu engagieren. Vgl. Green European Foundation: The European Citizens' Initiative Handbook, 2010, S. 88.

48 Protokoll (Nr. 1) über die Rolle der Nationalen Parlamente in der Europäischen Union, in: Amtsblatt der EU, Nr. C 83 vom 30. März 2010, S. 203-205.

49 Protokoll (Nr. 2) über die Anwendung der Grundsätze der Subsidiarität und der Verhältnismäßigkeit, in: Amtsblatt der EU, Nr. C 83 vom 30. März 2010, S. 206-209. 
fahrungen könnten durchaus auch mit Blick auf das Instrument der EBI genutzt werden, wobei politische Fraktionen Hauptakteure sein dürften.

Wenn es um transnationale politische Kommunikation geht, kommt heute automatisch das Internet als Plattform ins Blickfeld. Das Instrument der EBI bietet den potenziellen Nutzern dieser Plattform ein reiches neues Betätigungsfeld. Für die Mobilisierung einzelner Bürger - nicht zuletzt solchen, die nicht in fest gefügte Organisationen eingebunden sind hat die Plattform des Internets sicherlich viel Potenzial. Aber auch etablierte Interessenverbände, Organisationen der Zivilgesellschaft sowie politische Parteien auf allen Ebenen nutzen die neue Plattform fast schon routinemäßig.

\section{Fazit und Ausblick}

Die Überlegungen zur Nutzung und möglichen Wirkung der EBI haben ergeben, dass nur sehr vorsichtige Aussagen über die mögliche Wirkung dieses erstmals in den Verträgen verankerten direktdemokratischen Instruments möglich sind. Eher restriktiv dürften sich Faktoren auswirken, auf die in ganz generellen Überlegungen zu direktdemokratischen Mitwirkungs- und Entscheidungsformen immer wieder hingewiesen wird. Restriktive Wirkung dürfte, zweitens, eine Reihe von Details zur Ausgestaltung des neuen Instruments EBI einmal im Vertrag selbst, zum zweiten in der EBI-Verordnung - haben.

Sieht man hingegen das Neue und Innovative der Institution EBI darin, dass sie transnationale politische Kommunikation voraussetzt und anstößt - dass es also primär um die Beschaffung von (zusätzlicher), input'-Legitimation, nicht jedoch um die Herbeiführung konkreter inhaltlicher Politikergebnisse geht -, dann dürfte die Einschätzung der möglichen Wirkung der EBI positiver ausfallen. Bereits jetzt lässt sich beobachten, dass sich eine ganze Reihe von politischen Akteuren auf die Nutzung des neuen Instruments einstellen; sicher nicht ,blauäugig', sondern im vollen Bewusstsein um die ganz praktischen Schwierigkeiten. ${ }^{50}$ Wenn in einer Handreichung, die einen erfolgreichen Start des neuen Instruments fördern will, sehr detailliert zehn Punkte genannt und erläutert werden, die von Initiatoren und Organisatoren beachtet werden sollten, ${ }^{51}$ dann lässt das auf sorgfältig und zugleich systematisch vorangetriebene Vorarbeiten schließen.

Interessant und erwähnenswert sind in diesem Zusammenhang auch zwei Stellungnahmen des Wirtschafts- und Sozialausschusses vom Mai und Juli 2010, in denen diese EU-Institution $^{52}$ ihre Absicht kundtut, sich für den Erfolg des neuen Instruments einzusetzen und beispielsweise einen eigenen Beitrag zur Schaffung infrastruktureller Voraussetzungen - die Einrichtung einer Kontaktstelle, die konkrete Informationen und Hilfestellung geben soll zu leisten. ${ }^{53}$ Ein anderes Beispiel ist die Absicht der Regierung des Landes Salzburg, ein Büro einzurichten, welches potenziellen Initiatoren einer EBI Hilfestellung anbietet. ${ }^{54}$

Die EBI-Verordnung legt fest, dass sie ab 1. April 2012 gilt. Das heißt, dass ab diesem Datum Initiativen eingebracht werden können. Die dann einsetzende tatsächliche Nutzung des neuen Instruments wird sehr aufmerksam beobachtet und verfolgt werden. Die EBI-

50 Vgl. Green European Foundation: The European Citizens’ Initiative Handbook, 2010.

51 Ebenda, S. 77-86.

52 Sie ist im förmlichen EU-Entscheidungsprozess auf eine beratende Funktion beschränkt, beansprucht aber, zivilgesellschaftliche Kräfte zu repräsentieren und eine wichtige Mittlerfunktion zwischen ihnen und den EUInstitutionen wahrzunehmen, auch bei der Nutzung der EBI durch Organisationen der Zivilgesellschaft.

53 Vgl. Green European Foundation: The European Citizens' Initiative Handbook, 2010, S. 86-87.

54 Ebenda, S. 89. 
Verordnung schreibt vor, dass nach drei Jahren, also erstmals zum 1. April 2015, die Kommission dem Europäischen Parlament und dem Rat einen Bericht über die Anwendung der EBI-Verordnung vorlegen muss. Gestützt auf Erfahrungen, die während der ersten Jahre der Geltung der EBI-Verordnung gemacht werden, wird es dann eher möglich sein, besser fundierte Aussagen über die Wirkung der EBI als einer Innovation im Entscheidungssystem der Europäischen Union zu machen.

\section{Rhetorik des Politikerlobs}

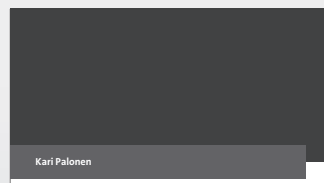

Rhetorik des Unbeliebten

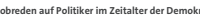

X Nomos

\section{Rhetorik des Unbeliebten}

Lobreden auf Politiker im

Zeitalter der Demokratie

Von Kari Palonen

2012, 209 S., brosch., 34,- $€$

ISBN 978-3-8329-7135-9

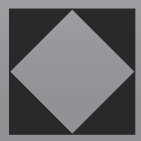

